\title{
Very long term observations of blazars - candidates for supermassive black hole binaries
}

\author{
Rene Hudec*t \\ Astronomical Institute of the Academy of Sciences of the Czech Republic \\ CZ-251 65 Ondrejov, Czech Republic \\ E-mail: rhudec@asu.cas.cz

\section{Milan Basta} \\ Astronomical Institute of the Academy of Sciences of the Czech Republic \\ CZ-251 65 Ondrejov, Czech Republic \\ E-mail: milan.basta@gmail.com
}

\begin{abstract}
The digitisation of astronomical plate archives, novel software and powerful computers allow data mining in extended plate databases for the first time. We refer on the project of searches for and analysis of supermassive binary black holes (binary blazars) requiring very long time intervals (50 years and more) which cannot be provided by other data sources. Examples of first preliminary data obtained from data mining in HCO plate stacks are presented.
\end{abstract}

Workshop on Blazar Variability across the Electromagnetic Spectrum

April 22-25, 2008

Palaiseau, France

* Speaker.

${ }^{\dagger}$ A footnote may follow. 


\section{Introduction}

The dominant fraction of the high energy celestial sources exhibit optical emission and hence can be also observed and investigated from the ground. These investigations are far less expensive than satellite measurements, and despite of this, can also provide valuable data. The multispectral approach has proved to be crucial in understanding of physical processes in observed objects. Although the large optical telescopes with CCDs and other advanced detectors can provide precise astrometry, photometry and spectroscopy of these sources, they are usually unable to provide long-time monitoring with good sampling. The real use of robotic telescopes is still limited and even these automated devices are usually unable to reach time coverage of order of ten years and more. However, the long-term monitoring of many of the celestial high-energy sources such as AGNs/blazars may be crucial for their understanding since they often shows long-term evolution, different activity states, brightness variations and/or flaring. This can be provided by data recorded in astronomical plate archives. The combination of data from several large plate collections can yield up to few tens of thousands of monitoring hours - one full life of an astronomer would be required to obtain the same observing every clear night by a CCD telescope. In the past, the data mining in astronomical plate archives was laborious and time-consuming, however the recent wide digitisation of the plates, as well as availability of powerful computers and novel software allow the effective data extraction for the first time.

Covering large time intervals is difficult but important task in astronomy and astrophysics. Archival plates represent a valuable tool in high-energy astrophysics especially by providing extended monitoring intervals with good sampling, allowing long-term evolution and changes to be studied, as well as detecting flares and other brightness variations. The photographic sky monitoring is available for more than 100 years. There are nearly 3 millions astronomical archival plates located at different observatories [9]. These archives can easily provide thousands of exposures for any celestial position, reaching monitoring intervals of up to few years of continuous monitoring i.e. tens of thousands of hours. Some of the archives have very high quality plates achieving limiting magnitudes of up to $20 \ldots 23$ (direct imaging) and /or 17...19 (spectral with objective prism). The recent efforts to digitise the plates and the corresponding software development significantly facilitate the extraction of unique scientific data from archival records and related reductions and analysis. Some of the archives already have devices for digitisation of plates and few of them have already started extended digitisation of the plates (e.g. Sonneberg Observatory - about 220000 plates already scanned). There are efforts to use these data for automated evaluation of objects on the plates and creating their light curves.

\section{Historical light curves and binary black holes in blazars}

In analogy to the most of other astrophysical branches the blazar science requires data to prove and build theory. Even though some features in blazars are rapid and often and thus in a short observational time theory can be built which can be confronted with the observations, other events, on the other hand, are long-term but at the same time fundamental for understanding the nature of blazars. These features are for example: Long-term periodicity in the light curve, detection of quiescent level, detection of large-amplitude flares etc. They may be induced by underlying physical 
processes such as: existence of supermassive binary black holes in blazars, stability, oscillations and changes of accretion disk, stability and changes in the jet etc. The study of the above mentioned features and processes requires either a good long-term coverage of the optical light curve in the history, or an excellent coverage of a specific period in the past. One of the interesting branches, that requires a good sampling of historical light curves, is the theory of supermassive binary black holes that are assumed to dwell in blazars. One of the most interesting blazars ever observed is the blazar called OJ 287, an easy target both for radio and optical telescopes. In 1988 Sillanpaa et al. [21] studied the optical light curve of this blazar and noticed a 12-year period within the major outbursts in the optical band of this blazar. The outbursts have a double peak structure with about one-year separation (the 12-year periodicity is neither strictly constant and nor is the separation of the peaks). Consequently, Sillanpaa et al. [21] predicted the next outburst to come in 1994. After the detection of the period in OJ 287, several models have been suggested to explain the behavior. Most of these models assume that the engine of OJ 287 is a pair of supermassive black holes (however also some non-binary black hole models have been suggested). Sillanpaa et al. [21] assume that the light variations are mass inflows from accretion disk into the black hole, caused by tidal force of the secondary black hole. In the Lehto and Valtonen model [15] the outbursts are associated with the companion black hole crossing of the accretion disk of the primary. Other models followed - e.g. Sundelius et al. [23] modeled the behavior of OJ 287 starting from the Lehto and Valtonen model, Katz [11] assumes that the secondary black hole exerts a torque on the accretion disk of the primary and thus causes the relativistic jet to sweep across our line of sight, Villata et al. [25] came up with a lighthouse model with a pair of bent jets. One of the last established and worked out model was published by Valtonen et al. [24]. In this model the features in the light curve are caused by 3 main processes: impacts of the secondary on the accretion disk of the primary, precession of the relativistic jet and tidal influence of the secondary on the accretion disk of the primary. The double-peak giant flares occurring with a 12-year period are associated with impacts of the secondary black hole on the accretion disk of the primary. The long-term 60-year trend observed in the light curve results from the precession of the relativistic jet, which is caused by the influence of the secondary on the accretion disk of the primary. The tidal influence of the secondary on the accretion disk of the primary causes variable accretion rate and thus a variable mass flow rate into the jet and a tidal outburst.Giant flares in 1947.3, 1973.0, 1983.0, 1984.2 and 1994.8 are used to construct a unique orbit solution. In this solution the binary precesses by 33 degrees per revolution - the precession is caused by relativistic precession and leads to loss of orbital energy due to gravitational radiation, the redshifted orbital period is 12.07 years, mass of the primary $=1.77 \times 10^{10}$ solar masses, mass of the secondary $=1.3 \times 10^{8}$ solar masses, major axis of the orbit $=0.056 \mathrm{pc}$, eccentricity $=0.70$. The inclination of the orbit is difficult to constrain with this model but the steep rise of outbursts led to conclusions that the orbital plane is nearly perpendicular to the plane of the accretion disk. The next giant flares were predicted [24]. The newly recognized and previously unknown giant flare of the blazar in 1956 found by one of us (RH) on Sonneberg archival sky patrol plates was used for the precise prediction within the binary precesion model. Moreover, theoretical reflections suggest that supermassive binary black holes may be common in the Universe. Direct and indirect observations show that supermassive black holes should be present in the center of nearby galaxies. In the bottom up scenario of galaxy formation supermassive binary black holes should then result from galaxy mergers of galaxies which 
each harbor a supermassive black hole. The estimated number of supermassive binary black holes should be relevantly high (e.g. [27]) which seems to be in contrast with observations. The explanation is that the orbital periods induced by the binary nature are of order of thousands of years, which are timescales that are out of any observational records. Fortunately, in the mass ratio/period plane there is a small region (large mass ratio-short orbital periods) where binary black holes can be detected through periodic behavior [29]. Blazars seem to be good candidates for detection of such a binary motion. We want to search for blazars that fit this region through historical light curves gathering and their consequent analysis.

We expect to find more blazars similar to a well-established supermassive binary black hole - OJ 287. We have gathered data from the literature and observational campaigns in order to establish long-term optical light curves of the below listed blazars-binary black hole candidates - to study periodic behavior in their light curves and other interesting features (intense outbursts, flares, quiescent level behavior). However, there are several crucial data gaps that disable to confirm periodicity or a BBH model (that has already been built up for several of these blazars). Therefore we intend to go to databases of astronomical plates to fill in these gaps. The most valuable databases are: Sonneberg Observatory, Germany (about 280,000 plates), Harvard College Observatory, USA (about 500,000 plates),UKSTU plate collection ROE Edinburgh, UK (18,000 very deep plates), and Observatory Leiden, NL (40,000 plates).

\section{Selection of binary black hole candidates}

As mentioned above, the first part of the study is the selection of suitable candidates. Based on extended literature and data search, we have selected almost a dozen of blazars as binary nature candidates for which we plan to gather the historical data points. The selected blazars were chosen by the following criteria: (i) known or suspected periodicities in their light curves, (ii) covered by archival plates and be within their magnitude limit, and (iii) Well-known blazars are preferred as the gathered data can be used more widely by the scientific community. The selected blazars are:

Mkn 501: This object was chosen because it is a well-known TeV blazar, which shows several possible indications for a binary-black hole nature [19], [26]. B 14 - 16 mag.

PKS 0420-014: This source is variable in many bands, it exhibits 13 -month variability between major outbursts [28] and its behavior has also been explained in the frame of a binary black hole model e.g. [4]. B 15.5 - 19 mag in 1989 - 1994.

Mrk 421: It is the brightest BL Lac object at the UV, X-ray and a well-known TeV gamma-ray source. Optical data for this source have also been provided by many observational campaigns. Its optical light curve suggests a possible 23-year periodicity [14]. B 11.6 up to 16 mag.

Mrk 766: An interesting X-ray source with possible X-ray periodicity [3]. This object was added to our list to study possible relations of periodicities in the X-ray and optical band.

ON 231: This object has been known as the variable ŞstarT since the beginning of the 20th century. In the optical band it exhibits variability on timescale of hours to years. There is a suggestion of a 13.6-year period in the optical band [13]. B = 13 mag in 1998 outburst, otherwise $\sim 14-$ 17 mag in the historical light curve. 
S2 0109+22: This object shows rapid optical variations of 2.5 mag in less than one year [6] and possible variations of base-level flux on a timescale of about 11.6 years [22]. B $\sim 14-17 \mathrm{mag}$ between $1994-2000$.

3C66A: 275 and 64-day periodicities were observed in the optical band [16],[12] found strong evidence for a 2.5-year period for the time interval of the moderate activity (1972-1992). B 1416 mag.

AO 0235+16: One of the most variable blazars across the entire electromagnetic spectrum. Suggestions for a 5.7-year periodicity [18] in the radio light curve and 2.95-year periodicity (Fan et al., 2002) in the optical light curve have also been interpreted as due to a binary black hole nature [20]; [17]. B 15-20 mag in the historical light curve.

3C 279: It is one of the best-monitored blazar sources. 3C 279 is an OVV with large and rapid outbursts. A strong period of 7.1 years was found (with Jurkevich method) in the long-term (27 years) near-infrared light curve [7]. Moreover, Abraham and Carrara [1], based on analysis of trajectories and velocities of superluminal features, got the best fit of a 22-year period. B $\sim 13.5-18$ mag between 1989-2004.

3C 345: Exhibits extreme variability in all wavelengths. Optical periodicities of 5 and 11 years have been reported followed up by binary black holes models [5].

S5 0716+71: A bright object in the optical band. The ejection of VLBA components in this source may be quasi-periodic occurring every 0.7 years [10].

For some of the above listed blazars the detected periods have been explained by the binary black hole nature: precessing jet (e.g. [20] for AO 0235+16; [5] for 3C 345), emission from the less massive binary black hole (e.g. [19] for Mkn 501), helically distorted jets ([26] for Mkn 501) etc.

\section{HCO Data Mining}

The first part of plate analyses at Harvard College Observatory (HCO) was focused on the data mining in the plate stacks with the intention to gather new historical data points for 9 blazars:

(1) OJ287 ( 550 recorded data points), (2) 3c66a ( 400 recorded data points), (3) S20109+22 ( $\sim 450$ recorded data points), (4) S50716+71 ( $\sim 450$ recorded data points), (5) ON231 ( $\sim 500$ recorded data points), (6) Mrk421 ( $\sim 550$ recorded data points), (7) PKS2155-304 ( $\sim 550$ recorded data points), (8) 3C371 ( 250 recorded data points), and (9) BL Lac ( $\sim 150$ recorded data points). Examples of preliminary very long-term (about 100 years) light curves based on recent HCO data mining are presented in Figs. 2 and 3. Note the preiliminary nature of these curves, as we still work on better calobration of used comparison stars which can considerably improve the measurement accuracy. It should be also noted that for a non-negligible fraction of the sources their magnitudes were measured on the plates with plate limits comparable to the brightness of the target.

\section{Conclusion}

Within the project described briefly in this paper, we intend to reach the following results: (i) improve historical light curves of candidates, (ii) periodicity and light curve analysis, (ii) confront the new light curve of the selected blazars with the corresponding theories, (iii) establish a detailed 
Figure 1: Left: The scan of the JD $=2419780.784$ plate, i.e. plate taken around the maximum of the 1913 outburst. Right: The V band light curve of OJ287 1913 outburst combining old (crosses) and new (filled circles) HCO plate measurements.
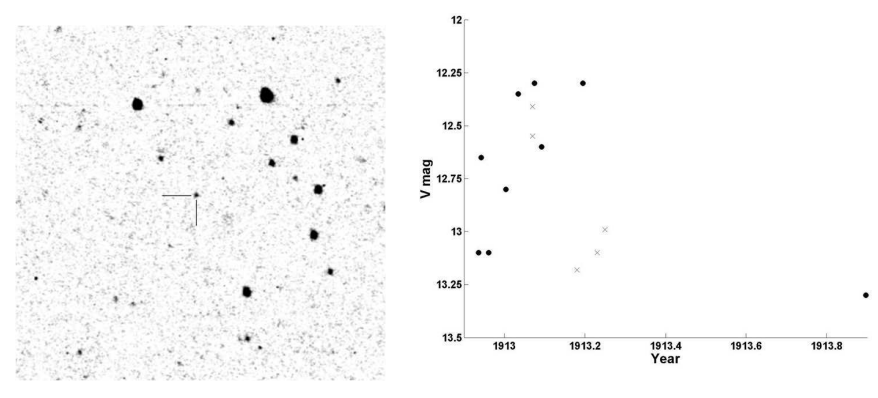

Figure 2: Left: OJ287. One new, previously unknown, historical outburst in 1900 added. The 2nd historical outburst in 1913 much better covered than before. Right: 3C371. 4 flaring episodes revealed over 100 years, amplitude $\sim 2 \mathrm{mag}$
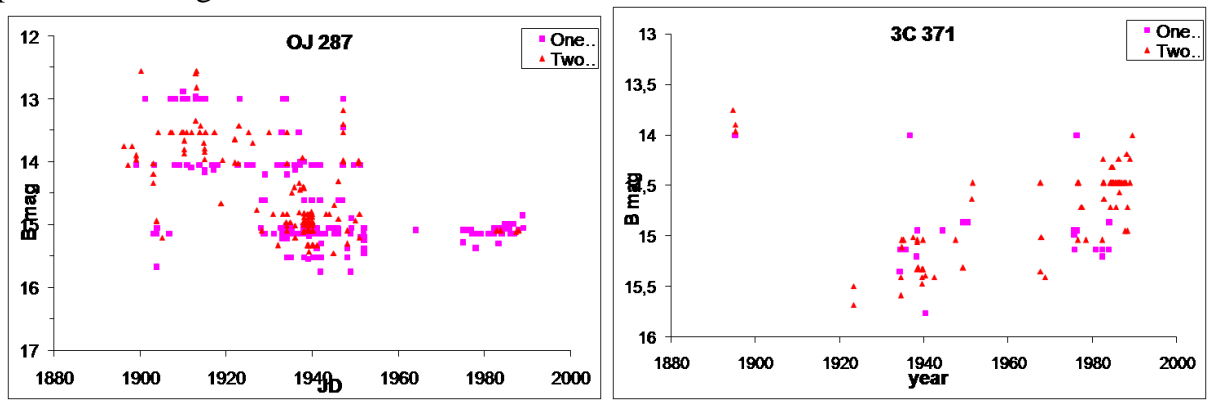

Figure 3: Left: 3C66a: Several flaring episodes, amplitudes $\sim 2-3$ mag, Right: Mrk 521, 7 sharp large flaring episodes revealed with fast rise and decline, one with large $(\sim 1.5 \mathrm{mag})$ amplitude
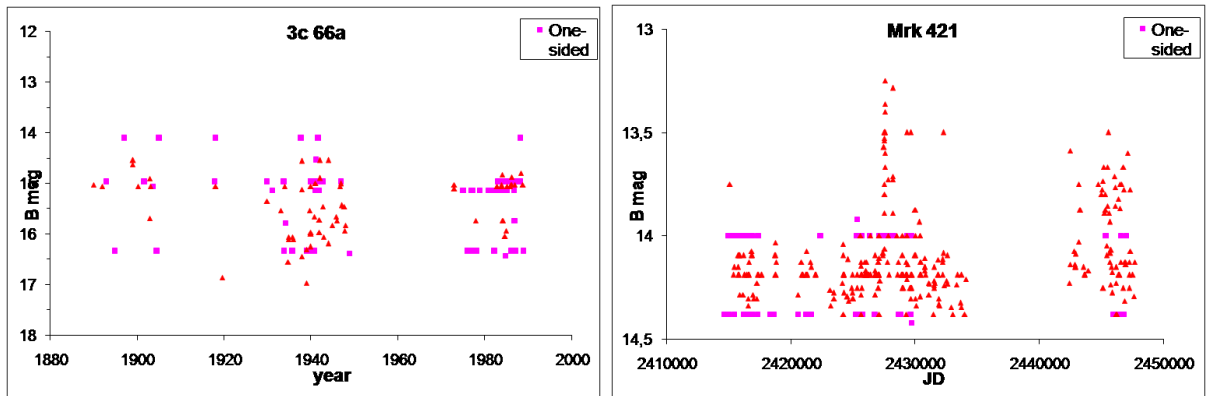

model at least of one of the candidates, (iv) draw statistical conclusions, (v) provide the data to wide scientific community. The nature of the project requires the availability of data sets covering very long time intervals, hence digitized archival plates are the obvious choice. The preliminary results presented in this paper confirm that this is useful approach, as the data are unique and cannot be provided by alternative methods.

\section{Acknowledgements}

We acknowledge the support by the Grant Agency of the Czech republic, grant 205/08/1207, 
and by ESA PECS 98023. We thanks to SAO and HCO for their support during our stay at HCO.

\section{References}

[1] Abraham, Z. \& Carrara, E. A., 1998, ApJ, 496, 172

[2] Babadzhanyants, M. K. \& Belokon, E. T., HE blazar astronomy, ASP Conf. Ser., 2003, 299

[3] Boller, Th. et al., 2001, A\&A, 365, 146

[4] Britzen, S. et al., 2000, A\&A, 360, 65

[5] Caproni, A. \& Abraham, Z., 2004, ApJ, 602, 625

[6] Ciprini, S. et al., 2003, A\&A, 400, 487

[7] Fan, J.H., 1999, MNRAS, 308, 1032

[8] Fan, J. H. et al., 2002, A\&A, 381, 1

[9] Hudec, R., 1999, Acta Historica Astronomiae, 6, 127.

[10] Jorstad, S. G. et al., 2001, ApJS, 134, 181

[11] Katz, J. I., 1997, ApJ, 478, 527

[12] Lainela, M. et al., 1999, ApJ, 521, 561

[13] Liu, F. K. et al., 1995, A\&A, 295, 1

[14] Liu, F. K. et al., 1997, A\&AS, 123, 569

[15] Lehto, H. J. \& Valtonen, M. J., 1996, ApJ, 460, 207

[16] Marchenko, S. G. et al., BL Lac Phenomenon, ASP Conference Series, 1999, 159

[17] Ostorero, L. et al., 2004, A\&A, 419, 913

[18] Raiteri, C. M. et al., 2001, A\&A, 377, 396

[19] Rieger, F. M. \& Mannheim, K., 2003, A\&A, 397, 121

[20] Romero, G. E. et al., 2003, astro-ph/0312197

[21] Sillanpaa, A. et al., 1988 ApJ, 325, 628

[22] Smith, A. G. \& Nair, A. D., 1995, PASP, 107, 863

[23] Sundelius, B. et al., 1997, ApJ, 484, 180

[24] Valtonen, M. J. et al., 2005, ApJ, submitted.

[25] Villata, M. et al., 1998, MNRAS, 293, L13

[26] Villata, M. \& Raiteri, C. M., 1999, A\&A, 347, 30

[27] Volonteri, M. et al., 2003, ApJ, 582, 559

[28] Wagner, S. J. et al., 1995, A\&A, 298, 688

[29] Yu, Q., 2002, MNRAS, 331, 935 